\title{
Pre-stroke warfarin enhancement of collateralization in acute ischemic stroke: a retrospective study
}

\author{
Jiaying Zhu ${ }^{1,2+}$, Mengmeng Ma ${ }^{1+}$, Yijia Guo ${ }^{1}$, Muke Zhou', Jian Guo ${ }^{1}$ and Li He ${ }^{1 *}$ (D)
}

\begin{abstract}
Background: Warfarin therapies not only are used to prevent stroke in patients with high risk of cardioembolism such as patients with atrial fibrillation (AF) and rheumatic heart disease (RHD), but also was associated with lower stroke severity and more favorable functional outcomes in patients with acute ischemic stroke due to middle cerebral artery occlusion. It was speculated that pre-stroke warfarin may promote collateralization and result in reduced stroke severity. This study aimed to investigate the association between pre-stroke warfarin use and leptomeningeal collaterals in patients with acute ischemic stroke due to occlusion of the middle cerebral artery.

Methods: We enrolled consecutive acute ischemic stroke patients (occlusion of the middle cerebral artery within $24 \mathrm{~h}$ ) with known history of AF and/or RHD at the neurology department of the West China Hospital from May 2011 to April 2017. Computed tomography angiography (CTA) before treatment was used to detect the thrombus. Regional leptomeningeal collateral (rLMC) score based on CTA images was used to assess collateral circulation. Prior use of warfarin was recorded. Univariate and multivariate analyses were performed to detect the association of prior warfarin use with the collateral circulation.
\end{abstract}

Results: A total of 120 patients were included; 29 (24.2\%) were taking warfarin before stroke. The international normalized ratio (INR) in patients with prior warfarin use was $1.53 \pm 1.00$, compared with $1.02 \pm 0.09$ in patients without prior warfarin use $(P<0.001)$. Prior oral warfarin therapy was inversely associated with poor $r \mathrm{LMC}(\mathrm{OR}=0$. $07,95 \% \mathrm{Cl} 0.01-0.44, P=0.005)$. There were no associations between prior warfarin use and initial stroke severity or functional outcomes at 3 months.

Conclusion: Warfarin use seems improve collateralization in patients with acute stroke. However, clinical controlled studies should be used to verify this claim.

Keywords: Warfarin, Acute ischemic stroke, Collateral circulation

\section{Background}

Leptomeningeal collaterals are pre-existing anastomoses of distal regions of intracerebral small blood vessels $[1,2]$. Collaterals provide the brain tissue with supplementary blood flow in the case of occlusion of primary intracranial arteries to protect brain tissue against irreversible damage [2]. Patients with good leptomeningeal collaterals usually have lower severity of stroke, better response to therapy, and better clinical

\footnotetext{
* Correspondence: heli2003new@126.com

${ }^{\dagger}$ Jia Ying Zhu and Mengmeng Ma contributed equally to this work.

'Department of Neurology, West China Hospital, Sichuan University,

Chengdu, China

Full list of author information is available at the end of the article
}

outcome in acute ischemic stroke [3-6]. Collateral blood vessels could be a therapeutic target in acute ischemic stroke [2]. However, preexisting collaterals depend on cardiac and pulmonary illnesses, metabolic syndrome, hyperuricemia, aging, and genetic factors [2, 4, 7, 8]. Although there are no pharmacologic treatments proven to promote cerebral collateralization currently, some conventional stroke treatments may be available to enhance the collateral circulation [6]. Statins, which have modest antithrombotic pleiotropic effects, have been shown to be associated with the presence of better collaterals in patients with acute stroke [9].

(C) The Author(s). 2018 Open Access This article is distributed under the terms of the Creative Commons Attribution 4.0 International License (http://creativecommons.org/licenses/by/4.0/), which permits unrestricted use, distribution, and 
In addition, numerous studies have demonstrated that oral anticoagulants (such as warfarin) reduced the risk of ischemic stroke [10]. Warfarin was also associated with lower stroke severity and more favorable functional outcomes when stroke occurs [11-13]. Although the mechanism for pre-stroke warfarin lowering stroke severity and improving functional outcomes was not very clear, two hypothesizes have been suggested. First, pre-stroke warfarin use may prevent thrombus formation and reduce the size of thrombi. Smaller thrombi cause distal infarctions and lower stroke severity and a more favorable clinical outcome [14, 15]. Secondly, pre-stroke warfarin use may promote collateralization since that the patients who used warfarin before stroke had lower stroke severity comparing with not using warfarin even though the patients of two groups had the similar thrombi and arterial occlusion site [16, 17]. Then it was speculated that pre-stroke warfarin may promote collateralization and result in reduced stroke severity. However, whether the association exit between pre-stroke warfarin use and collaterals still needs to be clarified. In this study, we aimed to evaluate the association between pre-stroke warfarin use and collaterals in acute ischemic stroke patients presenting with occlusion of the middle cerebral artery.

\section{Methods}

\section{Study population}

We performed a retrospective analysis of demographic, clinical, and radiographic data in consecutive patients at the Neurology Department of West China Hospital between May 2011 and April 2017. The inclusion criteria were: (1) $\geq 18$ years of age; (2) history of AF and/or RHD; (3) available computed tomography angiography (CTA) images within $24 \mathrm{~h}$ of symptom onset; and (4) CTA detection of acute infarction due to occlusion of the M1 or M2 middle cerebral artery (MCA), with or without occlusion of the internal carotid artery (ICA). The exclusion criteria were: (1) pre-stroke modified Rankin Scale (mRS) score $>2$; (2) severe extracranial vascular stenosis detected by CTA; or (3) < 90 days of follow-up.

\section{Admission $\mathrm{CT}$}

All patients underwent non-contrast computed tomography $(\mathrm{NCCT})$ and $\mathrm{CTA}$. Imaging was conducted on a 128-slice Siemens SOMATOM Definition Flash double source CT scanner. NCCT helical scans were performed form the skull base to the vertex using the following parameters: $70 \mathrm{kV}, 150 \mathrm{~mA}$, and 5-mm collimation. CTA was performed using: $42 \mathrm{ml}$ of contrast at $6 \mathrm{ml} / \mathrm{s}, 3$ to $5 \mathrm{~s}$ delay from injection to scanning, $70 \mathrm{kV}$, $150 \mathrm{~mA}, 0.5 \mathrm{~s} /$ rotation, and $1.25-\mathrm{mm}$ thick slice. The CTA scan covered the carotid bifurcation to the vertex.
Maximum-intensity projection images $(20 \mathrm{~mm})$ were reconstructed in the axial, sagittal, and coronal planes.

\section{Leptomeningeal collateral flow assessment}

We assessed the degree of leptomeningeal collateral flow using the regional leptomeningeal collateral (rLMC) score (20 points) [3]. The rLMC score is based on scoring the pial and lenticulostriate arteries in six MCA regions (M1-M6) and in the anterior cerebral artery region and the basal ganglia $(0=$ no; 1 = less; 2 = equal or more prominent compared with a matching region in the pial artery of the contralateral hemisphere). The arteries in the Sylvian sulcus are given a higher score: $0=$ not seen; $2=$ less; $4=$ same or more prominent compared with the opposite Sylvian sulcus. An rLMC score of $0-10$ was defined as poor collateral flow, 11-16 was defined as moderate collateral flow, and 17-20 was defined as good collateral flow [3]. The assessment was performed by four radiologists with extensive experience in acute stroke imaging. The average score was used as the final score for the analyses.

\section{Data collection}

Clinical variables (age, gender, stroke risk factors, admission examinations, previous medications, thrombolytic therapy, and hemorrhagic transformation) were collected for each patient from the stroke database. All biochemical indexes were measured within $24 \mathrm{~h}$ of admission. The patient was considered to be using warfarin if they had been using it for at least 3 months prior to stroke and were still using warfarin when stroke occurred. Neurological severity was assessed by trained neurologists using the National Institutes of Health Stroke Scale (NIHSS, < 5 was defined as mild stroke, $5-14$ as moderate stroke, and $>14$ as severe stroke) $[11,18]$. Intracerebral hemorrhage $(\mathrm{ICH})$ was diagnosed according to the European Cooperative Acute Stroke Study (ECASS) criteria. All patients were managed optimally according to the current guidelines, physicians' experience, and patients' comorbidities. Follow-up mRS at 3-month after discharge was used to assess the functional outcome. All data collectors were blind to the baseline collateral status.

\section{Statistical analysis}

Statistical analysis was performed using SPSS 22.0 for Windows (IBM, Armonk, NY, USA). The patients were grouped as warfarin users vs. non-warfarin users. The potential determinants of collaterals evaluated based on previous reports in the literature were age, blood pressure, total cholesterol (treated as continuous variables), high-density lipoprotein cholesterol (HDL-C), and low-density lipoprotein cholesterol (LDL-C), and history of stroke, TIA, hypertension, diabetes, hypercholesterolemia, and pre-stroke statin use (treated as 
dichotomized variables). [19, 20] Continuous variables were expressed as means \pm standard deviation or medians (interquartile range) and were analyzed using the Student's t test or the Mann-Whitney U test between two groups. When comparing percent distributions, the chi-square test was used but Fisher's exact test was used if the expected number in a category was $<5$. Univariate logistic regression was used to determine the association between potential determinants and leptomeningeal collaterals and multivariate logistic regression was used to determine the association between pre-stroke warfarin use and collaterals. Model 1 was adjusted for age, gender, onset to door time; model 2 included model 1 and was additionally adjusted for stroke risk factors; model 3 included model 2 and was additionally adjusted for admission examinations, thrombus location, NIH stroke scale scores; model 4 included model 3 and was additionally adjusted for prior medications. Logistic regression was used to determine the clinical outcomes associated with prestroke warfarin use. $P$ value $<0.05$ was considered statistically significant.

\section{Results}

\section{Baseline characteristics of the patients}

A total of 120 participants were included in the study. Of them, $37(30.8 \%)$ patients were male. Table 1 provided the characteristics of patients with pre-stroke warfarin use or not. Pre-stroke warfarin use was recorded in 29 (24.2\%) patients. Compared to patients without warfarin use, those with warfarin use were more likely to be younger age $(P<0.001)$ and to have a higher international normalized ratio (INR) $(\mathrm{P}<0.001)$.

\section{Assessment of clinical factors associated with collateral status}

Table 2 shows the association between clinical variables and rLMC scores (categorized as poor, moderate, and good). Previous coronary heart disease $(P=0.023)$, low-density lipoprotein cholesterol $(P=0.008)$, NIHSS $(\mathrm{P}<0.001)$, thrombus location $(\mathrm{P}<0.001)$, statin use $(P=0.068)$, and warfarin use $(P=0.045)$ were potential clinical factors associated with collateral flow. In the multivariate models (Table 3 ), prior warfarin use was inversely associated with poor $\mathrm{rLMC}(\mathrm{OR}=0.07$, 95\%CI 0.01-0.44, $P=0.005$ ) after adjustment for confounding factors.

\section{Association of Prior Warfarin use with stroke severity, Thrombus location, and clinical outcomes}

Table 4 shows the association between prior warfarin use and initial stroke severity, thrombus location, and functional outcome. No significant difference was observed between prior warfarin use and stroke severity or follow-up mRS. Compared with patients without warfarin use exposure, thrombus location (M2 vs. ICA + M1/M1) was significantly different in warfarin users (OR $=0.38,95 \% \mathrm{CI} 0.16-0.91, P=0.03)$. After adjustment for age, gender, previous antiplatelet and statins, warfarin users had a tendency to have distal vessel occlusion $(\mathrm{OR}=0.37,95 \% \mathrm{CI} 0.13-1.03, P=0.056)$.

\section{Discussion}

We performed a retrospective analysis of demographic, clinical, and radiographic data in consecutive patients with acute ischemic stroke presenting with the occlusion of middle cerebral artery to evaluate the association between pre-stroke warfarin use and collaterals by CTA images [21]. We found that pre-stroke oral warfarin use was inversely associated with poor leptomeningeal collaterals as identified. It showed that pre-stroke warfarin use may improve the poor cerebral collateral grade among acute ischemic stroke patients presenting with a major arterial occlusion by CTA images which is in accord with the hypothesis $[10,13]$.

This result also demonstrates a previously unidentified mechanism by which warfarin could lower the risks of poor collateral and reduce stroke severity. It was shown when intracranial artery occludes, secondary microthrombus may form in the distal small blood vessels of occluded artery $[10,15]$. This will interfere with collateral circulations in the small vessels. Consequently, warfarin may enhance or facilitate collaterals by 1 ) preventing the secondary microthrombus formation in the distal small blood vessels of occluded artery, 2) promoting the lysis of thrombi in the distal small vessels and speeding up the resolution process of these thrombi at stroke onset.

The international normalized ratio (INR) value has confirmed an inverse association between admission INR and infarct size in acute ischemic stroke [11, 2224]. In patients with higher INR (eg, an INR of 2-3), thrombus formation will be prevented and the resolution process of the new thrombus will be accelerated [25]. Indeed, one study conducted in Japan demonstrated that INR controlled between 1.60 and 1.99 is not effective for reducing the severity of ischemic stroke in patients with non-valvular atrial fibrillation [22]. Another study performed in the United States showed that therapeutic anticoagulation (INR $\geq 2$ ) was associated with lower odds of moderate or severe stroke [11]. In this study, the INR values of the patients with pre-stroke warfarin were relatively lower. This may explain the result of this study that collateral enhancement was only in the patients with poor rLMC compared with other patients. Therefore, it may be needed to explore the underlying effect of the INR on collaterals and stroke severity in the future. 
Table 1 Clinical characteristics of all participants $(n=120)$

\begin{tabular}{|c|c|c|c|c|}
\hline & All $(n=120)$ & Pre-stroke warf & & $P$ value \\
\hline & & Yes $(n=29)$ & No $(n=91)$ & \\
\hline Demographics, n (\%) & & & & \\
\hline Age $>60$ years & $89(74.2)$ & $12(41.4)$ & 77 (84.6) & $<0.001$ \\
\hline Male & $37(30.8)$ & $8(27.6)$ & 29 (31.9) & 0.664 \\
\hline Onset to door time $<6 \mathrm{~h}$ & $80(66.7)$ & 17 (58.6) & $63(69.2)$ & 0.291 \\
\hline Risk factors, n (\%) & & & & \\
\hline Hypertension & $60(50.0)$ & $10(34.5)$ & $50(54.9)$ & 0.055 \\
\hline Diabetes & $16(13.3)$ & $3(10.3)$ & $13(14.3)$ & 0.759 \\
\hline Atrial fibrillation & $112(93.3)$ & $22(75.9)$ & $90(98.9)$ & $<0.001$ \\
\hline Coronary heart disease & $17(14.2)$ & $3(10.3)$ & $14(15.4)$ & 0.76 \\
\hline Previous stroke & $16(13.3)$ & $2(6.9)$ & $14(15.4)$ & 0.352 \\
\hline Admission examinations, mear & & & & \\
\hline INR & $1.15 \pm 0.54$ & $1.53 \pm 1.00$ & $1.02 \pm 0.09$ & $<0.001$ \\
\hline Systolic blood pressure & $136.88 \pm 20.08$ & $133.86 \pm 23.74$ & $137.84 \pm 18.81$ & 0.356 \\
\hline Diastolic blood pressure & $81.31 \pm 15.03$ & $82.93 \pm 13.70$ & $80.79 \pm 15.47$ & 0.507 \\
\hline Serum glucose & $7.85 \pm 2.40$ & $7.45 \pm 2.71$ & $7.97 \pm 2.29$ & 0.311 \\
\hline Triglyceride & $1.53 \pm 1.60$ & $1.90 \pm 2.65$ & $1.40 \pm 1.08$ & 0.72 \\
\hline Cholesterol & $3.97 \pm 0.99$ & $3.91 \pm 1.29$ & $3.98 \pm 0.88$ & 0.325 \\
\hline $\mathrm{HDL}-\mathrm{C}$ & $1.38 \pm 0.56$ & $1.36 \pm 0.79$ & $1.39 \pm 0.47$ & 0.26 \\
\hline LDL-C & $2.25 \pm 0.72$ & $2.16 \pm 0.65$ & $2.29 \pm 0.74$ & 0.402 \\
\hline Clinical factors, n (\%) & & & & \\
\hline Thrombus location on CTA & & & & 0.078 \\
\hline $\mathrm{ICA}+\mathrm{M} 1$ & $28(23.3)$ & $6(20.7)$ & $22(24.2)$ & \\
\hline M1 & $54(45.0)$ & $9(31.0)$ & $45(49.5)$ & \\
\hline M2 & $38(31.7)$ & $14(48.3)$ & $24(26.3)$ & \\
\hline NIHSS scores & $13(7-18)$ & $12(8-16)$ & $13(7-18)$ & \\
\hline Thrombolysis & $22(18.3)$ & $4(13.8)$ & $18(19.8)$ & 0.468 \\
\hline Intracranial hemorrhage & $27(22.5)$ & $5(17.2)$ & $22(24.2)$ & 0.436 \\
\hline Pre-stroke medications, n (\%) & & & & \\
\hline Antihypertensive drugs & $49(40.8)$ & $10(34.5)$ & $39(42.9)$ & 0.424 \\
\hline Antidiabetics & $14(11.7)$ & $3(10.3)$ & $11(12.1)$ & 0.549 \\
\hline Antiplatelet drugs & $21(17.5)$ & $2(6.90)$ & 19 (20.9) & 0.084 \\
\hline Statins & $21(17.5)$ & $7(24.1)$ & $14(15.4)$ & 0.28 \\
\hline
\end{tabular}

INR international normalized ratio, $H D L$ high density lipoprotein, $L D L$ high density lipoprotein, CTA computer tomography angiography; ICA internal carotid artery, NIHSS National Institutes of Health Stroke Scale

Previous studies have confirmed that vitamin $\mathrm{K}$ antagonists, such as warfarin, or non-vitamin antagonist oral anticoagulants (NOACs) reduce the risk of ischemic stroke [26]. Additionally, the current guidelines recommend warfarin or NOACs for stroke prevention in high-risk patients with AF or RHD. Nevertheless, many patients still fail to receive proper stroke prevention in the real world. A previous study showed that only $61.8 \%$ outpatients with AF (CHADS2 score $\geq 2$ ) were treated with warfarin or NOACs [27]. In this study, we found that only $29(31.9 \%)$ patients were treated with warfarin, and therefore the treatment rate is far below the rates observed in western countries. In addition, current guidelines suggest that a target INR between 2.0 to 3.0 is considered as effective [28]. Because of the high risk of intracranial hemorrhage in the Asian population, Japanese guidelines recommend that patients $>70$ years of age should be controlled with a target INR of 1.60-2.60 [29]. In the United States, a large, nationwide and registry study indicated that in patients with acute ischemic stroke and a known history of $\mathrm{AF}, 30 \%$ were receiving some form of oral 
Table 2 Associated factors with collateral circulation in acute ischemic stroke patients

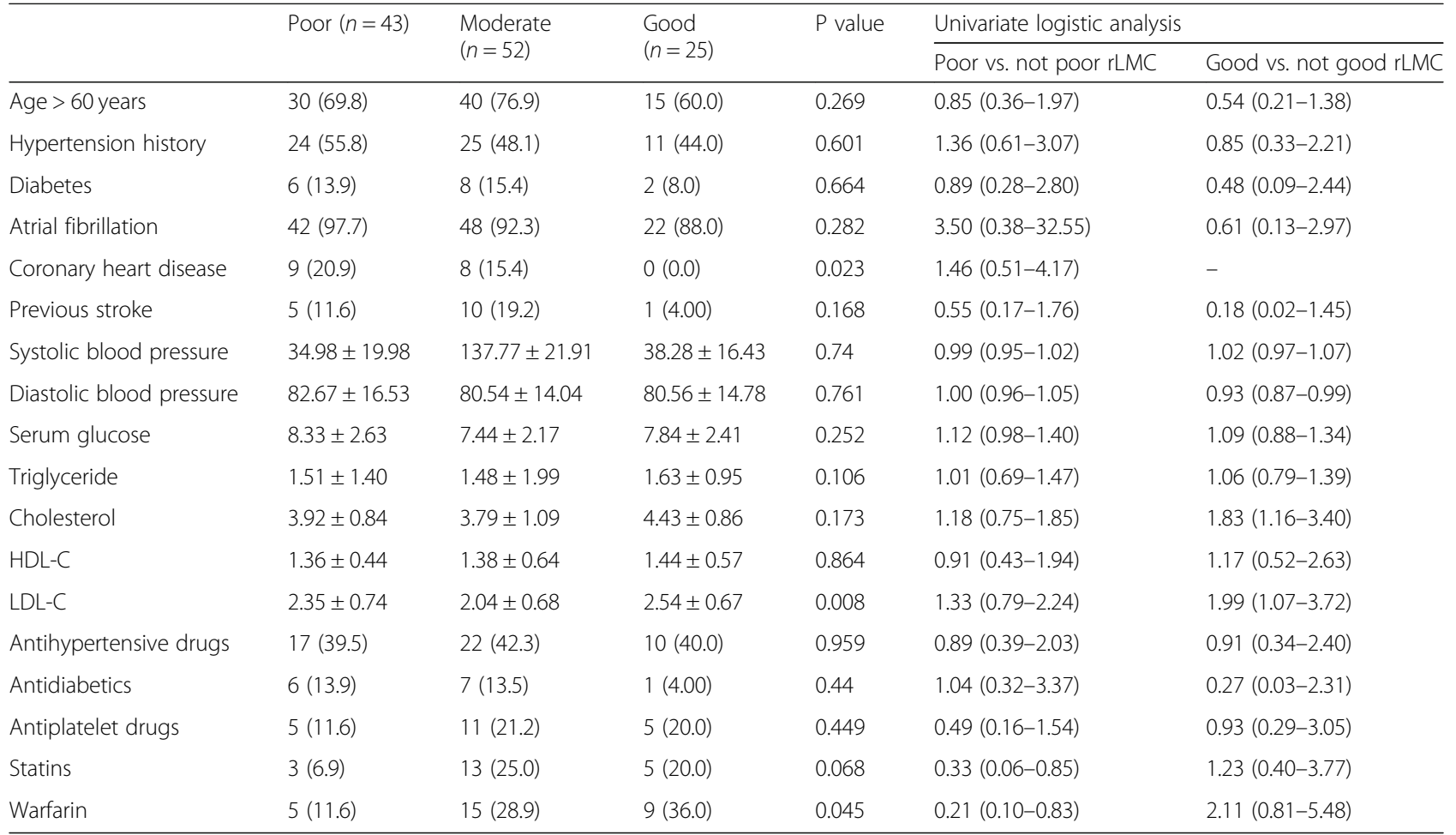

HDL high density lipoprotein, LDL high density lipoprotein, CTA computer tomography angiography, ICA internal carotid artery, NIHSS National Institutes of Health Stroke Scale, LMC leptomeningeal collateral

antithrombotic therapy before stroke onset, but that $64 \%$ of the warfarin-treated patients were receiving sub-therapeutic doses [11].

The presence of collateral cerebral circulation has been shown to be associated with better functional outcomes after stroke [3-6]. After acute ischemic stroke onset, collateral vessels provide the brain tissue with supplementary blood flow. Guidelines are available regarding the methods clinically available or under trial to promote collateral circulation after stroke (head position, induced hypertension, volume expansion, external

Table 3 Association between pre-stroke warfarin use and collaterals

\begin{tabular}{|c|c|c|c|c|}
\hline & \multicolumn{2}{|l|}{$\begin{array}{l}\text { Poor rLMC vs. } \\
\text { Not poor rLMC }\end{array}$} & \multicolumn{2}{|l|}{$\begin{array}{l}\text { Good rLMC vs. } \\
\text { Not good rLMC }\end{array}$} \\
\hline & OR $(95 \% \mathrm{Cl})$ & $P$ value & OR $(95 \% \mathrm{Cl})$ & $P$ value \\
\hline Unadjusted & $0.21(0.10-0.83)$ & 0.021 & $2.11(0.81-5.84)$ & 0.125 \\
\hline Model 1 & $0.18(0.05-0.61)$ & 0.006 & $1.92(0.66-5.62)$ & 0.234 \\
\hline Model 2 & $0.17(0.05-0.65)$ & 0.009 & $2.12(0.61-7.39)$ & 0.239 \\
\hline Model 3 & $0.06(0.01-0.37)$ & 0.002 & $6.03(0.94-38.71)$ & 0.058 \\
\hline Model 4 & $0.07(0.01-0.44)$ & 0.005 & $4.80(0.68-33.79)$ & 0.115 \\
\hline
\end{tabular}

Model 1: Adjusted for age, gender, onset to door time; Model 2: Adjusted model 1 plus stroke risk factors;

Model 3: Adjusted model 2 plus admission examinations, thrombus location, $\mathrm{NIH}$ stroke scale scores;

Model 4: Adjusted model 3 plus prior medications counter pulsation, bypass surgery, albumin, nitric oxide, TNF- $\alpha$ inhibitors, statins [6]); but these methods are examined in the context of stroke management, not prevention. Nevertheless, some factors are known to be associated with collaterals. Previous studies have shown negative associations between hypertension and collaterals, and positive associations between statin use and collaterals [5, 9]. In addition, age, ischemic preconditioning, and cardiovascular risk factors are considered to be associated with collateral circulation patency [3, 30, 31]. Particularly, metabolic syndrome, high blood uric acid levels, and age have been associated with poor collaterals [4]. In the present study, the multivariable analysis showed that pre-stroke oral warfarin use was associated with collaterals, independently of age, gender, onset to door time, stroke risk factors, admission examinations, thrombus location, $\mathrm{NIH}$ stroke scale scores, and pre-stroke medications (model 4). This may be one reason why pre-stroke warfarin use affects stroke severity and clinical outcome. Nevertheless, additional studies in a larger population are necessary to corroborate our observations.

The major challenge for antithrombotic therapy among patients with AF or RHD is the risk of bleeding. In the present study, $22.5 \%$ of the patients with acute ischemic stroke were detected with $\mathrm{ICH}$. A previous 
Table 4 Association between pre-stroke warfarin use and clinical outcomes

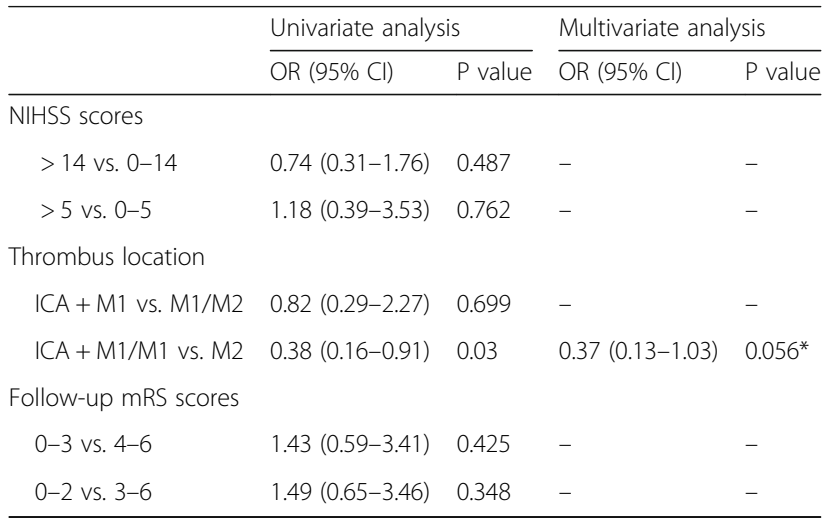

${ }^{*}$ Adjusted age, gender, previous antiplatelet and statins, NIHSS National Institutes of Health Stroke Scale

ICA internal carotid artery, mRS modified Rankin Scale

report indicated that anticoagulant treatment after stroke onset was associated with significant increase in symptomatic intracranial bleeding [32], but we did not find that pre-stroke warfarin use was associated with $\mathrm{ICH}$, which is supported by a previous study [13]. Nevertheless, due to the relatively small sample size in the present study, interpretation should be made with caution. There are several limitations to our study. First, the sample size was relatively small and we failed to perform subgroup analyses; hence, potential heterogeneity exists in the study population. Secondly, this study did not control for the use of previous antiplatelet therapy. Finally, based on the INR, most patients in the present study were found to receive inadequate antithrombotic therapy.

\section{Conclusions}

The results showed that prior warfarin use was inversely associated with the risk for poor collaterals in acute ischemic stroke patients presenting with occlusion of the middle cerebral artery, suggesting that warfarin use seems improve collateralization in patients with acute stroke. However, clinical controlled studies should be used to verify this claim.

\footnotetext{
Abbreviations

AF: Atrial fibrillation; CT: Computer tomography; CTA: Computer tomography angiography; ECASS: European Cooperative Acute Stroke Study; HDL: High density lipoprotein; ICA: Internal carotid artery; ICH: Intracerebral hemorrhage; INR: International normalized ratio; LDL: High density lipoprotein; MCA: Middle cerebral artery; MRI: Magnetic resonance imaging; mRS: Modified Rankin Scale; NCCT: Non-contrast computer tomography; NIHSS: National Institutes of Health Stroke Scale; NOACs: Non-vitamin antagonist oral anticoagulants; OR: Odds ratio; RHD: Rheumatic heart disease; rLMC: Regional leptomeningeal collateral; SD: Standard difference
}

\section{Acknowledgments}

We thank all the participants for their participation in this study.

\section{Funding}

This work was supported by National Comprehensive Demonstration Research on Prevention and Control of Chronic Diseases in Southwest China (2018YFC1311400 and 2018YFC1311401).

\section{Availability of data and materials}

The datasets used and analyzed in the current study are not publicly available. Anonymous data is available from the corresponding author upon reasonable request.

\section{Authors' contributions}

$J Z$ and MM analyzed and interpreted the patient data and were the main contributors in writing the manuscript. They contributed equally to this work. YG performed the statistically analysis and was major contributor in writing the manuscript. MZ and JG collected the data and were the major contributors in writing the manuscript.

LH participated in the study design, data interpretation and revising the manuscript critically for important intellectual content. All authors read and approved the final manuscript.

\section{Ethics approval and consent to participate}

The Ethics Committee of West China Hospital, Sichuan University approved the study protocol. All patients consent to participate in this observational study. Written informed consent or oral consent was obtained from each participant according to the demand of Local Ethics Committee of West China Hospital, Sichuan University. The ethics committee approved this procedure. The dataset was anonymized prior to analysis.

Consent for publication

Not applicable.

\section{Competing interests}

The authors declare that they have no competing interests.

\section{Publisher's Note}

Springer Nature remains neutral with regard to jurisdictional claims in published maps and institutional affiliations.

\section{Author details}

${ }^{1}$ Department of Neurology, West China Hospital, Sichuan University, Chengdu, China. 'Department of Emergency, Gui Zhou provincial People's Hospital, Guiyang, China.

Received: 30 July 2018 Accepted: 19 November 2018 Published online: 29 November 2018

\section{References}

1. Liebeskind DS. Collateral circulation. Stroke. 2003;34(9):2279-84. https://doi. org/10.1161/01.str.0000086465.41263.06 Epub 2003/07/26. PubMed PMID: 12881609 .

2. Shuaib A, Butcher K, Mohammad AA, Saqqur M, Liebeskind DS. Collateral blood vessels in acute ischaemic stroke: a potential therapeutic target. Lancet Neurol. 2011;10(10):909-21. https://doi.org/10.1016/s14744422(11)70195-8.

3. Menon BK, Smith EE, Modi J, Patel SK, Bhatia R, Watson TW, et al. Regional leptomeningeal score on $C T$ angiography predicts clinical and imaging outcomes in patients with acute anterior circulation occlusions. AJNR Am J Neuroradiol. 2011;32(9):1640-5. https://doi.org/10.3174/ajnr.A2564 PubMed PMID: 21799045.

4. Menon BK, Smith EE, Coutts SB, Welsh DG, Faber JE, Goyal M, et al. Leptomeningeal collaterals are associated with modifiable metabolic risk factors. Ann Neurol. 2013;74(2):241-8. https://doi.org/10.1002/ana.23906 PubMed PMID: 23536377; PubMed Central PMCID: PMC3836863.

5. Lima FO, Furie KL, Silva GS, Lev MH, Camargo EC, Singhal AB, et al. The pattern of leptomeningeal collaterals on CT angiography is a strong predictor of long-term functional outcome in stroke patients with large vessel intracranial occlusion. Stroke. 2010;41(10):2316-22. https://doi.org/10. 1161/STROKEAHA.110.592303 PubMed PMID: 20829514; PubMed Central PMCID: PMC4939434.

6. Bang OY, Goyal M, Liebeskind DS. Collateral circulation in ischemic stroke: assessment tools and therapeutic strategies. Stroke 2015;46(11):3302-3309. 
doi: https://doi.org/10.1161/STROKEAHA.115.010508. PubMed PMID: 26451027; PubMed Central PMCID: PMC4624512.

7. Teunissen PF, Horrevoets AJ, van Royen N. The coronary collateral circulation: genetic and environmental determinants in experimental models and humans. J Mol Cell Cardiol. 2012;52(4):897-904. https://doi.org/ 10.1016/.yjmcc.2011.09.010 PubMed PMID: 21959171.

8. Epstein SE, Lassance-Soares RM, Faber JE, Burnett MS. Effects of aging on the collateral circulation, and therapeutic implications. Circulation. 2012; 125(25):3211-9. https://doi.org/10.1161/CIRCULATIONAHA.111.079038 PubMed PMID: 22733335.

9. Ovbiagele B, Saver JL, Starkman S, Kim D, Ali LK, Jahan R, et al. Statin enhancement of collateralization in acute stroke. Neurology. 2007;68(24): 2129-31. https://doi.org/10.1212/01.wnl.0000264931.34941.f0 PubMed PMID: 17562834.

10. Hylek EM, Skates SJ, Sheehan MA, Singer DE. An analysis of the lowest effective intensity of prophylactic anticoagulation for patients with nonrheumatic atrial fibrillation. N Engl J Med. 1996;335(8):540-6. https://doi.org/10.1056/ nejm199608223350802 Epub 1996/08/22 PubMed PMID: 8678931.

11. Xian Y, O'Brien EC, Liang L, Xu H, Schwamm LH, Fonarow GC, et al. Association of Preceding Antithrombotic Treatment with Acute Ischemic Stroke Severity and in-Hospital Outcomes among Patients with Atrial Fibrillation. JAMA. 2017;317(10):1057-67. https://doi.org/10.1001/jama.2017. 1371 PubMed PMID: 28291892.

12. Tziomalos K, Giampatzis V, Bouziana SD, Spanou M, Kostaki S, Papadopoulou $M$, et al. Adequacy of preadmission oral anticoagulation with vitamin $K$ antagonists and ischemic stroke severity and outcome in patients with atrial fibrillation. J Thromb Thrombolysis. 2016;41(2):336-42. https://doi.org/10. 1007/s11239-015-1262-y PubMed PMID: 26253707.

13. Audebert HJ, Schenk B, Schenkel J, Heuschmann PU. Impact of prestroke oral anticoagulation on severity and outcome of ischemic and hemorrhagic stroke in patients with atrial fibrillation. Cerebrovasc Dis. 2010;29(5):476-83. https://doi.org/10.1159/000297963 PubMed PMID: 20299787.

14. Miteff FLC, Bateman GA, Spratt N, McElduff P, Parsons MW. The independent predictive utility of computed tomography angiographic collateral status in acute ischaemic stroke. Brain. 2009;132(Pt 8):2231-8. https://doi.org/10.1093/brain/awp155.

15. YM WM, Minematsu K, Yamaguchi T. Effects of anticoagulation on infarct size and clinical outcome in acute cardioembolic stroke. Angiology. 2002;53(5):551-6

16. DeFazio RALS, Morales CL, Levy RV, Dave KR, Lin HW, Abaffy T, Watson BD, Perez-Pinzon MA, Ohanna V. A protocol for characterizing the impact of collateral fl ow after middle cerebral artery occlusion. Transl Stroke Res. 2011;2(1):112-27.

17. Yuan FLX, Guan Y, Mu Z, Chen K, Wang Y, Yang GY. Collateral circulation prevents masticatory muscle impairment in rat middle cerebral artery occlusion model. J Synchrotron Radiat. 2014;21(6):1314-8. https://doi.org/10. 1107/S1600577514016130 PubMed Central PMCID: PMCPMID: 25343800

18. Kvistad CE, Logallo N, Oygarden H, Thomassen L, Waje-Andreassen U, Naess $H$. Elevated admission blood pressure and stroke severity in acute ischemic stroke: the Bergen NORSTROKE study. Cerebrovasc Dis. 2013;36(5-6):351-4. https://doi.org/10.1159/000355685 PubMed PMID: 24192488.

19. Pourati IKC, Rand W, Karas RH. Statin use is associated with enhanced collateralization of severely diseased coronary arteries. Am Heart J. 2003; 146(5):876-81

20. Paciaroni M AG, Micheli S, Caso V. Efficacy and safety of anticoagulant treatment in acute cardioembolic stroke: a meta-analysis of randomized controlled trials. Stroke. 2007;38(2):423-430. Epub 430. doi: https:/doi.org/ 10.1161/01.STR.0000254600.92975.1f. PubMed Central PMCID: PMC17204681.

21. Chalothorn DFJ. Formation and maturation of the native collateral circulation. J Mol Cell Cardiol. 2010;49:251-9.

22. Matsumoto M, Sakaguchi M, Okazaki S, Hashikawa K, Takahashi T, Matsumoto $\mathrm{M}$, et al. Relationship between infarct volume and prothrombin time-international normalized ratio in ischemic stroke patients with Nonvalvular atrial fibrillation. Circ J. 2017;81(3):391-6. https://doi.org/10. 1253/circj.CJ-16-0707 PubMed PMID: 28154247.

23. Ay $H$, Arsava EM, Gungor $L$, Greer D, Singhal AB, Furie $K L$, et al. Admission international normalized ratio and acute infarct volume in ischemic stroke. Ann Neurol. 2008;64(5):499-506. https://doi.org/10.1002/ana.21456 PubMed PMID: 18661516.

24. McDowell TYL, Florian J, Southworth MR, Grant S, Stockbridge N. Relationship between international normalized ratio and outcomes in modern trials with warfarin controls. Pharmacotherapy. 2018. https://doi. org/10.1002/phar.2161.

25. Yasaka MMK, Yamaguchi T. Optimal intensity of international normalized ratio in warfarin therapy for secondary prevention of stroke in patients with non-valvular atrial fibrillation. Intern Med. 2001;40(12):1183-8.

26. Penttilä TLM, Niiranen J, Mehtälä J, Khanfir H, Lassila R, Raatikainen P. Differences in the risk of stroke, bleeding events, and mortality between female and male patients with atrial fibrillation during warfarin therapy. Eur Heart J Cardiovasc Pharmacother. 2018. https://doi.org/10.1093/ehjcvp/ pvy026 PubMed Central PMCID: PMCPMID: 30052822.

27. Hsu JC, Maddox TM, Kennedy K, Katz DF, Marzec LN, Lubitz SA, et al. Aspirin instead of Oral anticoagulant prescription in atrial fibrillation patients at risk for stroke. J Am Coll Cardiol 2016;67(25):2913-2923. Epub 2016/06/25. doi: https://doi.org/10.1016/j.jacc.2016.03.581. PubMed PMID: 27339487.

28. January CT, Wann LS, Alpert JS, Calkins H, Cigarroa JE, Cleveland JC, Jr., et al. 2014 AHA/ACC/HRS guideline for the management of patients with atrial fibrillation: executive summary: a report of the American College of Cardiology/American Heart Association task force on practice guidelines and the Heart Rhythm Society. Circulation 2014;130(23):2071-2104. Epub 2014/04/01. doi: https://doi.org/10.1161/cir.0000000000000040. PubMed PMID: 24682348.

29. Shinohara Y, Yamaguchi T. Outline of the Japanese guidelines for the Management of Stroke 2004 and subsequent revision. Int J Stroke 2008;3(1): 55-62. Epub 2008/08/19. doi: https://doi.org/10.1111/j.1747-4949.2008. 00178.x. PubMed PMID: 18705916.

30. Faber JE, Zhang H, Lassance-Soares RM, Prabhakar P, Najafi AH, Burnett MS, et al. Aging causes collateral rarefaction and increased severity of ischemic injury in multiple tissues. Arterioscler Thromb Vasc Biol. 2011;31(8):1748-56. https://doi.org/10.1161/ATVBAHA.111.227314 PubMed PMID: 21617137; PubMed Central PMCID: PMC3141082.

31. Wang J, Peng X, Lassance-Soares RM, Najafi AH, Alderman LO, Sood S, et al. Aging-induced collateral dysfunction: impaired responsiveness of collaterals and susceptibility to apoptosis via dysfunctional eNOS signaling. J Cardiovasc Transl Res. 2011;4(6):779-89. https://doi.org/10.1007/s12265-0119280-4 PubMed PMID: 21538183; PubMed Central PMCID: PMC3756560.

32. Paciaroni M, Agnelli G, Micheli S, Caso V. Efficacy and safety of anticoagulant treatment in acute cardioembolic stroke: a meta-analysis of randomized controlled trials. Stroke. 2007;38(2):423-30. https://doi.org/10. 1161/01.STR.0000254600.92975.1f PubMed PMID: 17204681.

\section{Ready to submit your research? Choose BMC and benefit from:}

- fast, convenient online submission

- thorough peer review by experienced researchers in your field

- rapid publication on acceptance

- support for research data, including large and complex data types

- gold Open Access which fosters wider collaboration and increased citations

- maximum visibility for your research: over $100 \mathrm{M}$ website views per year

At BMC, research is always in progress.

Learn more biomedcentral.com/submissions 